\title{
Printed instead of cultured
}

Nat. Commun. 8, 1327 (2017)

Microbial biophotovoltaic cells (BPVs) produce electricity using photosynthetic organisms such as cyanobacteria as the light harvester. These organisms have the advantage of being environmentally friendly and low cost, and have self-repair capabilities. However, the development of BPVs has met with many difficulties. In addition to the low power output, the inability to produce these on a commercial scale is another major concern. This is because existing BPVs use a bulky liquid culture reservoir, from which cyanobacteria are deposited onto electrode surfaces, and the sedimentation process is lengthy. Now, Peter Nixon and colleagues in the United Kingdom digitally print both the cyanobacteria and the electrode components with a commercial inkjet printer, removing the need to use the liquid reservoir and offering potentially improved scalability.

The researchers started by printing carbon nanotube (CNT) patterns on a paper substrate and subsequently on top of those conductive patterns, cyanobacteria. They then paired the printed bioelectrode (as an anode) with a platinized carbon cathode that is exposed to the air as in conventional BPVs. This hybrid BPV produces a power output of $0.38 \mathrm{mWm}^{-2}$ in the light and $0.22 \mathrm{mWm}^{-2}$ in the dark, comparable to conventional BPVs. In their second device, the researchers printed both the bioanode and a CNT cathode on a paper substrate, and then covered them with a hydrogel that serves as a salt bridge between the electrodes as well as a growth medium for the printed cyanobacteria. This thin-film BPV displays a power output stability of 100 hours during repeated light and dark cycles, although the power density is much lower than the hybrid BPV.

Changjun Zhang

Published online: 8 December 2017

https://doi.org/10.1038/s41560-017-0064-1 